\title{
THE
}

\section{In Situ Measuring Partition Coefficient at Intact Nanoemulsions: A New Application of Single-Entity Electrochemistry}

Hiranya Madawala

University of Rhode Island

Shashika Gunathilaka Sabaragamuwe

University of Rhode Island

Subhashini Elangovan

University of Rhode Island

Jiyeon Kim

University of Rhode Island, jkim25@uri.edu

Follow this and additional works at: https://digitalcommons.uri.edu/chm_facpubs

The University of Rhode Island Faculty have made this article openly available.

Please let us know how Open Access to this research benefits you.

This is a pre-publication author manuscript of the final, published article.

Terms of Use

This article is made available under the terms and conditions applicable towards Open Access

Policy Articles, as set forth in our Terms of Use.

\section{Citation/Publisher Attribution}

Madawala, H. ,Sabaragamuwe, S. G., Elangovan, S., \& Kim, J. (2021). In Situ Measuring Partition Coefficient at Intact Nanoemulsions: A New Application of Single-Entity Electrochemistry. Anal. Chem., 93(2), 1154-1160. doi: 10.1021/acs.analchem.0c04205

Available at: https://doi.org/10.1021/acs.analchem.0c04205 


\title{
In Situ Measuring Partition Coefficient at Intact Nanoemulsions: A New Application of Single Entity Electrochemistry
}

\author{
Hiranya Madawala $^{1}$, Shashika Gunathilaka Gunathilaka Sabaragamuwe ${ }^{1}$, Subhashini Elangovan ${ }^{1}$, \\ Jiyeon $\operatorname{Kim}^{1 *}$ \\ ${ }^{1 .}$ Department of Chemistry, University of Rhode Island, Kingston, RI, 02881 \\ E-mail: jkim25@uri.edu
}

\begin{abstract}
We report a new application of the single entity electrochemistry to in-situ measure a partition coefficient at intact nanoemulsions (NEs). The partition coefficient at intact NEs is the most crucial physicochemical property to determine the uptake of delivery molecules inside NEs. It, however, has not been unequivocally elucidated by currently existing techniques based on exsitu measurements. Herein, we apply the single entity electrochemistry (SEE) to directly and quantitatively measure the partition coefficient at NEs in situ. In this work, we use NEs featured with amphiphilic triblock copolymer (Pluronic F-127) as a model system to extract/preconcentrate 2-aminobiphenyl (2-ABP) dissolved in the water, and demonstrate a new application of SEE to in situ quantitatively estimate the amounts of 2-ABP distributed into each intact NE . Our SEE measurements reveal that the partitioning is governed by extraction of 2-ABP inside NEs rather than its adsorption on NE surface, and this extraction is remarkably efficient with up to $\sim 8$ orders of magnitude of the preconcentration factor, thus leading to the unprecedentedly large partition coefficient of $1.9( \pm 1.4) \times 10^{10}$. This result implies that not only the thermodynamic distribution but also the intermolecular interaction of extracted compounds inside NEs could play a significant role in the apparent partition coefficient $\left(P=1.9( \pm 1.4) \times 10^{10}\right)$. The experimentally determined partition coefficient was validated by molecular dynamics (MD) simulations with showing a stabilizing role of intermolecular interaction in the partitioned system. We further verified our methodology with other compounds exhibiting aromatic property, e.g., ferrocene methanol. Significantly, our new approach can be readily applicable to investigate practical NEs commercially marketed for drug, food, and cosmetics.
\end{abstract}




\section{Introduction}

Nanoemulsions (NEs) are oil-in-water (o/w) emulsions with 50 to $500 \mathrm{~nm}$ of mean droplet diameter. ${ }^{1}$ Particularly, this small size and the hydrophobic phase inside enable NEs to be a promising tactic for the efficient delivery of hydrophobic molecules e.g. drugs, food, or cosmetics, thus attracting NE applications in personal care, cosmetics and health care. ${ }^{1,2,3}$

This strategy strongly relies on a partition coefficient of target compounds at NEs, which is the crucial physicochemical property for their applications. The partition coefficient is defined as the ratio of delivery compounds distributed between the organic phase and aqueous phase under equilibrium in a delivery system. ${ }^{4}$ It determines the uptake or encapsulation of delivery compounds in NEs, ${ }^{5,6}$ and the capacity of NEs to dissolve large quantities of hydrophobes, along with their ability to protect these compounds from hydrolysis and degradation. ${ }^{1}$

In general, the partition coefficient of target compounds at NEs have been determined by common analytical techniques such as high performance liquid chromatography (HPLC), ${ }^{7,8}$ reverse-phase HPLC, ${ }^{9}, 10$ FTIR spectroscopy, ${ }^{11}$ UV-Vis spectrophotometry, ${ }^{12}$ or scanning electrochemical microscopy. ${ }^{13}$ These approaches, however, offer indirect and ex situ measurements, where the concentrated NEs are ultrafiltrated or centrifuged, then the resulting filtrate is analyzed by the corresponding techniques, or an alternative mimicking system is indirectly studied. ${ }^{7,8,13,14}$ Although these measurements provide an important insight about partition coefficient at NEs, the accuracy, precision, and the relevance of the determined value for intact NEs is equivocal. Even for the commercially marketed NEs, a partition coefficient is vaguely given based on these ex situ measurements. ${ }^{15}$ More importantly, understanding whether the partitioning is governed by extraction or adsorption phenomenon, is a prerequisite to fully utilize the extraction efficiency of NEs. The general ex situ approaches, however, cannot explicitly elucidate the partitioning process in NEs.

Herein, we demonstrate the first application of single entity electrochemistry (SEE) to in situ measure the partition coefficient at intact NEs at an individual level. In the earlier work, we have uniquely applied SEE to study the inner-structure of monodisperse NEs and the structural impact on NE's electrochemical behaviors, ${ }^{16}$ whereas SEE has been widely applied to polydisperse NEs to discretely characterize their size distribution as opposed to ensemble measurements. ${ }^{17}$ In this study, we select Pluronic F-127 functionalized NEs as a model system, which have been practically 
used for pharmaceutical, ${ }^{7}$ or photochemical applications, ${ }^{18,19}$ and investigate a partition coefficient of 2-aminobiphenyl (2-ABP) as a targeting hydrophobe at intact NEs. Particularly, our model NEs are highly monodisperse, which is more than adequate in this work, since responses in SEE measurements would depend on not the variations in size of NEs but the amount of partitioned molecules. Also, we interrogate current-time responses observed in real-time during SEE measurements to clarify the governing process of partitioning. Further, we perform the MD simulations to validate the experimentally determined partition coefficient at intact NEs as well as gain the molecular insight in the partitioned system. In the analytical point of view, the combination of SEE and NEs with high monodispersity could offer the high accuracy and precision in the present study.

\section{Experimental Section}

Chemicals. Pluronic F-127 (F-127), bis(2-ethylhexyl)-sebacate (castor oil, or DOS 97.0\%), tetrahydrofuran (THF, 99.9\%), 2-aminobiphenyl (2-ABP, 97\%), and potassium tetrakis[pentafluorophenyl] borate (KTFPB, 95\%) were used as obtained from Sigma-Aldrich. Also, ammonium hexafluorophosphate, $\mathrm{NH}_{4} \mathrm{PF}_{6}$ (99.98\%), ferrocenemethanol (FcMeOH, 97\%), ammonium phosphate monobasic (98\%) and ammonium phosphate dibasic (98\%) were purchased from Sigma-Aldrich and used as obtained. Nanopure water $(18.2 \mathrm{M} \Omega \cdot \mathrm{cm}$, TOC $2 \mathrm{ppb}$; Milli-Q Integral 5 system, Millipore) was used for both the preparation of aqueous electrolyte solutions and NE synthesis.

Solution preparation. $0.0017 \mathrm{~g}$ of 2 -ABP was dissolved in $2 \mathrm{~L}$ of nanopure water and $5 \mu \mathrm{M}$ stock solution was prepared. From 2-ABP stock solution, a series of diluted solutions ranging from 3 $\mu \mathrm{M}$ to $1 \mathrm{nM}$ were prepared. The aqueous solution contained $10 \mathrm{mM} \mathrm{NH}_{4} \mathrm{PF}_{6}$ with the $\mathrm{pH}$ adjusted to $\mathrm{pH} 7$ with $2 \mathrm{mM}$ ammonium monobasic phosphate and ammonium dibasic phosphate.

SEE Measurements. SEE was performed at ambient temperature $\left(20^{\circ} \mathrm{C}\right)$ with a two-electrode cell using a bipotentiostat (CHM8022D, CH Instrument, Austin, TX). Right before SEE measurements, electrolyte solutions with addition of NEs were vortexed at $1000 \mathrm{rpm}$ for $15 \mathrm{~min}$, and left on the lab bench for up to $2 \mathrm{hrs}$ to ensure homogeneous mixing. For SEE measurements, we immersed a Pt ultramicroelectrode (Pt UME) with $5 \mu \mathrm{m}$ diameter in the aqueous solution containing $10 \mathrm{mM} \mathrm{NH}_{4} \mathrm{PF}_{6}$ and $2 \mathrm{mM}$ phosphate buffer. The constant potential optimized to 0.85 
$\mathrm{V}$ vs the $\mathrm{Pt}$ quasi-reference electrode (Pt QRE) was applied for the amperometric $i-t$ measurements during SEE. The time interval for data acquisition was 5-10 ms.

More detailed information about synthesis of NEs, TEM measurements with the Uranyless negative staining method, ${ }^{16}$ and fabrication of Pt UMEs ${ }^{16}$ is in Supporting information (SI).

\section{Results and Discussions}

Practical Nanoemulsions for a Model System. We used NEs featured with amphiphilic triblock copolymers (Pluronic F-127) and DOS plasticizer. Similar composition of NEs has been employed in optical nanosensing ${ }^{18,19}$ or pharmaceutical extractor. ${ }^{7}$ The optimized synthesis of our NE attained the high monodispersity in size with $40( \pm 5) \mathrm{nm}$ diameter through the characterization by TEM (Figure 1B and 1C). High monodispersity observed in both low and high resolution TEM images is consistent with dynamic light scattering (DLS) measurements showing c.a. $38 \mathrm{~nm}$ diameter with $0.15( \pm 0.05)$ polydispersity index (PDI) (Figure S1). These highly monodisperse NEs are more than adequate in this work, since variations in responses of SEE measurements are mainly dependent on the variations in the amount of 2-ABP extracted in NEs rather than variations in size of NEs.

(A)

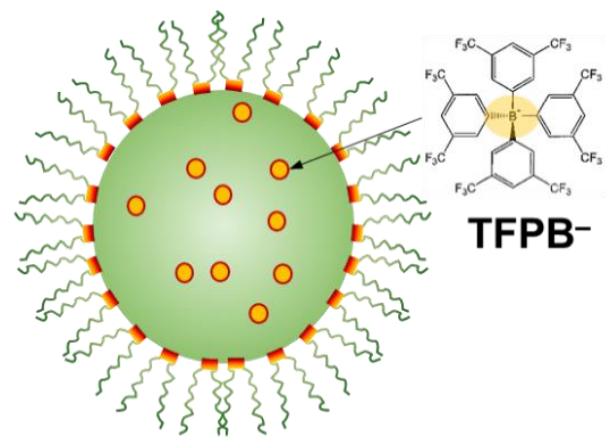

Pluronic F-127 based NEs
(B)

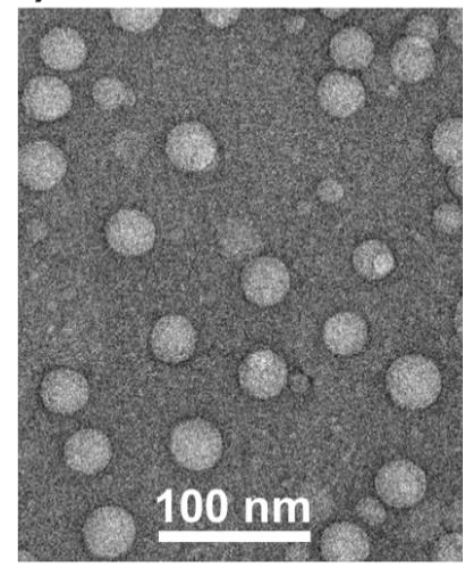

(C)

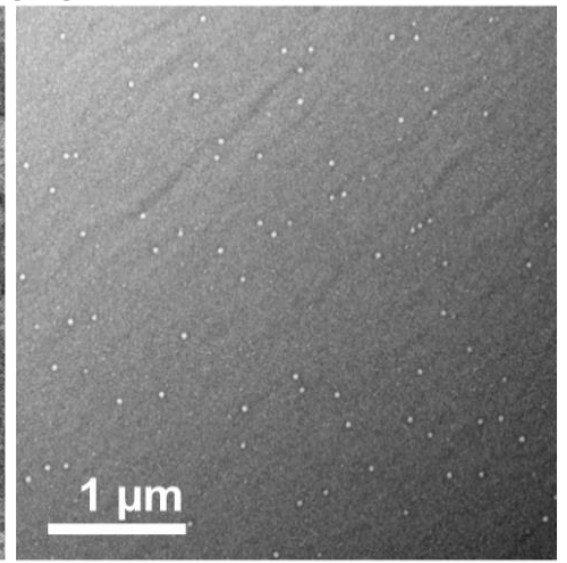

Figure 1. (A) Schematic illustration of Pluronic F-127 decorated NEs containing cation exchanger, TFPB $^{-}$and castor oil as plasticizer. (B), (C) TEM images at high and low resolutions, respectively. Notably, high monodispersity of NEs is observed over the wide range. 


\section{Single Entity Electrochemistry with Individual NEs Partitioned with 2-ABP from Aqueous}

Bulk Solution. To estimate a partition coefficient at given NEs, 2-ABP was used as target molecule. 2-ABP is highly hydrophobic with a large partition coefficient of 691.8 between octanol and water. ${ }^{20} 2$-ABP undergoes one electron transfer oxidation reaction to form cationic radical, often followed by polymerization reaction depending on the experimental condition (Figure S2 in SI), where the electropolymerization is triggered at extremely positive oxidation potential (see section 3 in SI). ${ }^{21,22}$ To avoid any complexity in our SEE measurements, an oxidation potential of 0.85 V vs. Pt QRE lower than $E_{1 / 2}$ (i.e. 1.10 V vs. Pt QRE) was selected.

In Figure 2, a scheme of SEE measurement is depicted, where a Pt UME applied with a constant potential of $0.85 \mathrm{~V}$ vs. Pt QRE is immersed in the aqueous solution containing both freshly prepared NEs and 2-ABP at a concentration lower than $\mu \mathrm{M}$. Right before the SEE measurements, we vortexed aqueous solutions for 15 min once NEs were added, and left on the benchtop for 2 hrs to ensure the system under the equilibrium as well as homogeneous mixing. In the aqueous bulk solution, 2-ABP concentration is low enough not to give any substantial background current in the current-time $(i-t)$ response during SEE measurements. NEs partitioned with 2-ABP spontaneously diffuse and collide onto a Pt UME, where an oxidative potential is applied. Upon this individual collision of a NE, 2-ABP partitioned in (or, at) a NE is immediately oxidized, thus giving anodic currents, which decay with time due to the depletion of 2-ABP in (or, at) a NE. Accordingly, a current spike is observed upon an individual collision of a NE. Further, the integration of a current spike over time yields charges needed for electrolysis of 2-ABP, i.e., an amount of 2-ABP partitioned in (or, at) a NE. Particularly, the high monodispersity of NEs allows us to consider their volume constant, and to estimate a concentration of 2-ABP in (or at) an individual NE. 


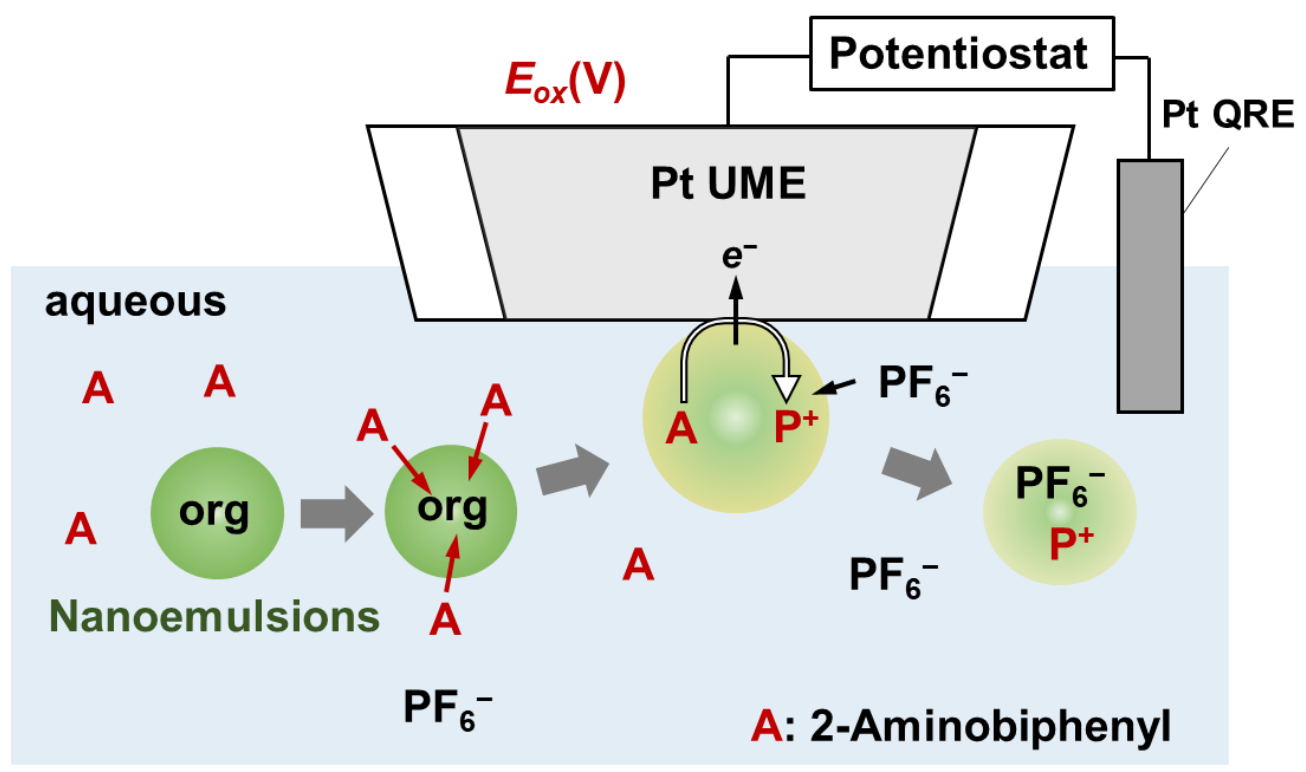

Figure 2. A scheme of SEE measurements. NEs partitioned with 2-ABP spontaneously diffuse in the aqueous bulk solution containing a low concentration of 2-ABP, and collide onto a Pt UME applied with a constant oxidative potential, $E_{o x}=0.85 \mathrm{~V}$ vs. Pt QRE. Upon a collision of an individual NE, 2-ABP partitioned in (or, at a NE) is electrolyzed leading to an anodic current flow. During this electrolysis, an anion, $\mathrm{PF}_{6}{ }^{-}$in aqueous phase is transferred into the $\mathrm{NE}$ to maintain the electroneutrality inside a NE. ${ }^{16}$ Aqueous solution contains $10 \mathrm{mM} \mathrm{NH}_{4} \mathrm{PF}_{6}$ as a supporting electrolyte.

Herein, we conducted three sets of SEE measurements under three different concentrations of 8.0 pM, 0.8 pM, or 80 fM NEs. In each set of SEE measurements, we varied the concentration of 2ABP in aqueous solution within three orders of magnitudes. The first set of SEE was performed under $8 \mathrm{pM}$ NEs with the concentration of $2-\mathrm{ABP}$ at from $5.0 \mu \mathrm{M}$ to $0.1 \mu \mathrm{M}$. For instance, with 8 $\mathrm{pM}$ of NEs and $5.0 \mu \mathrm{M} 2-\mathrm{ABP}$ in aqueous solution, we measured $i$ - $t$ curve showing a series of current spikes with nearly uniform current magnitudes. A typical $i$ - $t$ curve is illustrated in Figure 3A. Characteristically, each current spike showed an exponential decay with time. This collisional response, $i$ - $t$ decay was fitted with bulk electrolysis model, where we assumed that extracted 2ABP diffuses in DOS) inside a NE, and undergoes an electrolytic reaction through a contact point between a NE and a Pt UME ${ }^{16,17}$ (See section 5 and Figure S4 in SI for more details). In Figure 3A insets, a good agreement between the experimental $i$ - $t$ curve (black solid lines) and the simulation (red open circles) undoubtedly evidences that partitioned 2-ABP locates in a NE filled with DOS, not on the surface of a NE. Thereby, the partitioning of 2-ABP at NEs is mainly 
governed by the extraction process not the adsorption. Notably, in situ measurements by SEE uniquely prove this strong evidence of extraction as a governing process in the partitioning of delivery compounds at NEs, which cannot be explicitly answered by other ex situ techniques such as HPLC or spectrophotometry.

Continuously, we collected charges from integrated current spikes to construct a concentration distribution curve of 2-ABP partitioned in NEs. For this analysis, we only counted current spikes at least 3 times larger than the background noise signal for this analysis (Background $i$ - $t$ curve responses with blank samples are shown in Figure S5, SI). Note that SEE responses are independent with size of NEs due to the high monodispersity, thereby a variation of charges in SEE measurements is mainly caused by a variation of 2-ABP amount partitioned in a NE. Using a constant radius, $19 \mathrm{~nm}$ of monodisperse NEs $\left(r_{N E}\right)$ determined by TEM and DLS, and the integrated charge $(Q)$ from $i$ - $t$ curve, the concentration of $2-\mathrm{ABP}$ partitioned in a $\mathrm{NE}, C_{2-A B P}^{N E}$ is calculated by $^{17}$

$C_{2-A B P}^{N E}=\frac{3 Q}{4 \pi F r_{N E}^{3}}$

where $F$ denotes Faraday constant $(96,485 \mathrm{C} / \mathrm{mol})$.

The overall distribution of $C_{2-A B P}^{N E}$ estimated from current spikes (Figure 3A), is illustrated in Figure 3B. The resulting $C_{2-A B P}^{N E}$ ranges $14.0 \sim 17.0 \mathrm{M}$ with a peak at $15.5 \mathrm{M}$, which is consistent with the maximum capacity of given NEs, $15.8 \mathrm{M}$ for ferrocene determined by our previous work. ${ }^{16}$ In this earlier work of SEE measurements, the full electrolysis of $15.8 \mathrm{M}$ ferrocene inside NEs could be observed within c.a. $0.5 \mathrm{~s}$ of duration time as similar durations are observed in insets of Figure 3. Notably, a narrow distribution of $C_{2-A B P}^{N E}$ was obtained, which could be ascribed to not only monodisperse NEs but also the fully equilibrated system. This fully equilibrated system as well as the high monodispersity of NEs is beneficial to attain the high accuracy and precision in our analysis, since repetitive measurements can be made with similar NEs during each round of SEE measurements. This new analytical aspect is distinctive compared to the general scope of conventional SEE applications with polydisperse NEs limited to a discrete size distribution of NEs.

Further, we sequentially reduced 2 -ABP concentration in aqueous solution up to $0.1 \mu \mathrm{M}$ in the presence of 8 pM NEs, and subsequently conducted the SEE. The respective $C_{2-A B P}^{N E}$ values were 
estimated from each $i$ - $t$ curve, and the corresponding concentration distribution curves were constructed in the same manner as aforementioned (data not shown). Overall, as 2-ABP concentration in aqueous solution decreases from $5.0 \mu \mathrm{M}$ to $0.1 \mu \mathrm{M}$ under $8 \mathrm{pM} \mathrm{NEs}$, the $C_{2-A B P}^{N E}$ peak value linearly decreases from $15.5 \mathrm{M}$ to $0.5 \mathrm{M}$ (Figure 4 ). We will discuss more details in the later section.

Likewise, we conducted another sets of SEE in the presence of $0.8 \mathrm{pM}$ NEs with 2-ABP concentration from $1.0 \mu \mathrm{M}$ to $10 \mathrm{nM}$. In Figure 3C, a typical $i$ - $t$ curve with $0.8 \mathrm{pM}$ NEs and 0.3 $\mu \mathrm{M} 2-\mathrm{ABP}$ is depicted. The resulting current spikes followed well behaved bulk electrolysis model indicating a partitioning of 2-ABP via extraction inside a NE (insets in Figure 3C). Based on the charges integrated from each current spike and eq (1), the distribution curve of $C_{2-A B P}^{N E}$ was constructed, narrowly ranging between 12.0 and $18.0 \mathrm{M}$ with a peak at $15.5 \mathrm{M}$ (Figure 3D). Note that NEs could hold 2-ABP with a maximum capacity under this given condition as well. In addition, as the concentration of 2-ABP in aqueous solution sequentially decreases from $1.0 \mu \mathrm{M}$ to $10 \mathrm{nM}$ under $0.8 \mathrm{pM}$ NEs, a linear decrease in $C_{2-A B P}^{N E}$ peak values is observed ranging from 15.5 M to $0.5 \mathrm{M}$ similar to the case with $8 \mathrm{pM}$ NEs (Figure 4).

The final set of SEE measurements was performed under $80 \mathrm{fM}$ NEs with 2-ABP concentration at from $0.1 \mu \mathrm{M}$ to $1.0 \mathrm{nM}$. As shown in Figure 3E with $80 \mathrm{fM}$ NEs and $30 \mathrm{nM}$ 2-ABP, a characteristic $i-t$ curve is observed. Each current spike follows bulk electrolysis model implying a partitioning of 2-ABP via extraction inside a NE as well (insets in Figure 3E). The subsequent distribution curve of $C_{2-A B P}^{N E}$ shows a narrow width between 12.0 and $17.0 \mathrm{M}$ with a peak at $15.5 \mathrm{M}$ (Figure $3 \mathrm{~F}$ ). When 2-ABP concentration in aqueous solution reduced up to $1.0 \mathrm{nM}$ under $80 \mathrm{fM} \mathrm{NEs}$, the respective SEE measurements gave a subsequent $C_{2-A B P}^{N E}$ peak value, $0.9( \pm 0.4) \mathrm{M}$ (Figure S5 in $\mathrm{SI})$. Overall, a linear decrease in $C_{2-A B P}^{N E}$ peak values from $15.5 \mathrm{M}$ to $0.9 \mathrm{M}$ is also obtained, as the 2-ABP concentration in aqueous solution sequentially decreases from $0.1 \mu \mathrm{M}$ to $1.0 \mathrm{nM}$ under 80 fM NEs (Figure 4). 

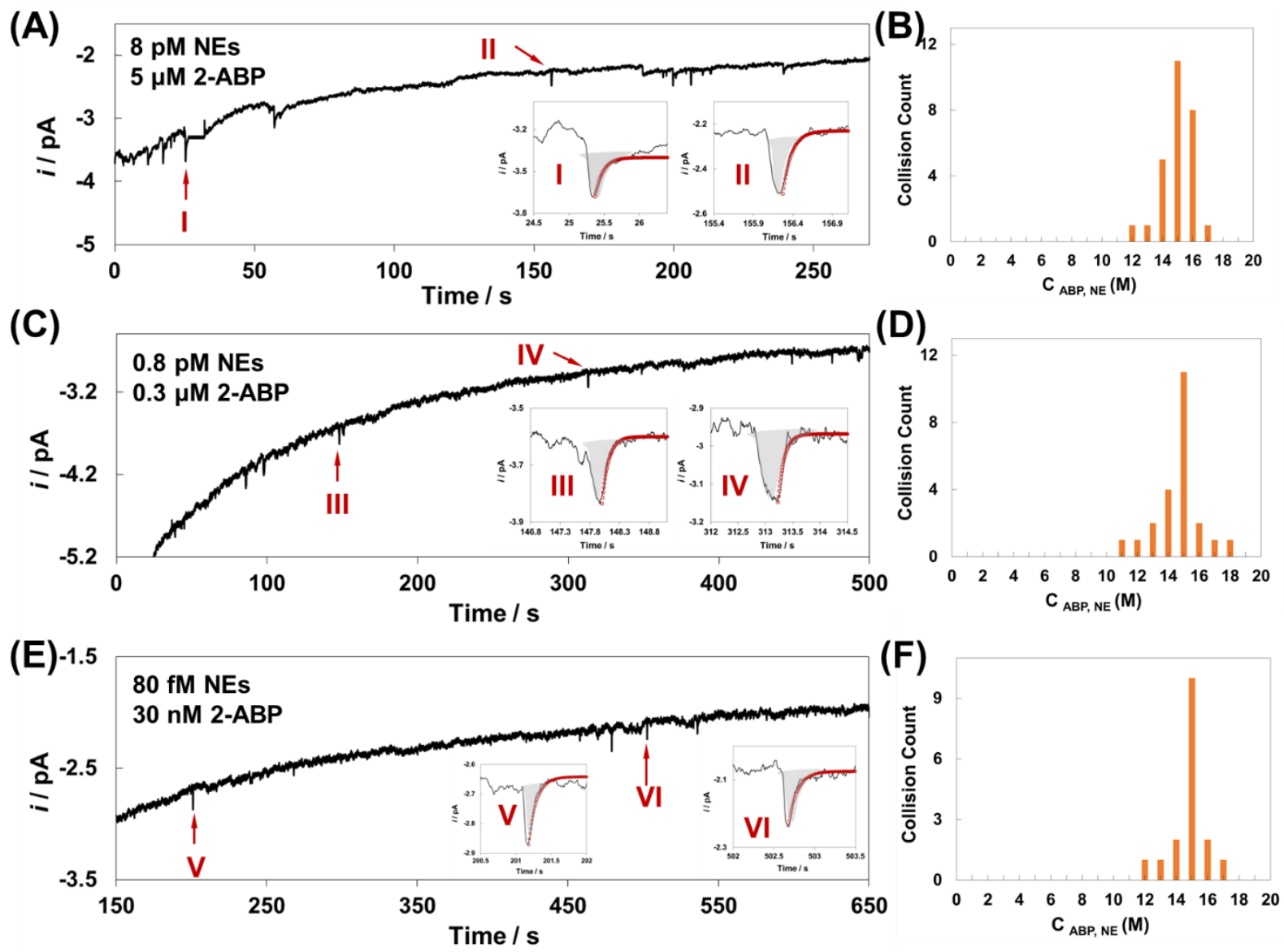

Figure 3. (A), (C), (E) $i$ - $t$ curves of NE collisions at Pt UME under $0.85 \mathrm{~V}$ vs Pt QRE with (8 pM $\mathrm{NEs}+5 \mu \mathrm{M} 2-\mathrm{ABP}),(0.8 \mathrm{pM} \mathrm{NEs}+0.3 \mu \mathrm{M} 2-\mathrm{ABP})$, and (80 fM NEs + $30 \mathrm{nM} 2-\mathrm{ABP})$, respectively. Each inset shows a comparison between experimental current spike (black solid lines) and simulated one (red open circles) based on the bulk electrolysis model. (B), (D), (F) Concentration distribution curves of 2-ABP partitioned in NEs, $\boldsymbol{C}_{\mathbf{2}-\boldsymbol{A B P}}^{\boldsymbol{N} \boldsymbol{P}}$ from the corresponding SEE data.

The Partition Coefficient at Intact NEs and Its Validation by the Molecular Dynamics Simulation. Finally, we could construct a plot of $C_{2-A B P}^{N E}$ vs. total concentration of 2-ABP, $C_{2-A B P}^{t o t}$ by collecting all the $C_{2-A B P}^{N E}$ peak values from three sets of SEE data with $8 \mathrm{pM}, 0.8 \mathrm{pM}$, and 08 pM NEs throughout analyzing 1500-2000 current spikes under each given condition (Figure 4). Three linear curves were obtained with respectively different slopes (determined by the least square regression). 
Here, we formulate the relationship between $C_{2-A B P}^{N E}$ and $C_{2-A B P}^{t o t}$ by considering the following equilibrium constant and the mass balances. When 2-ABP is partitioned from water to organic phase of DOS in NEs,

$2-A B P(a q) \rightleftarrows 2-A B P(N E)$

$P=\frac{C_{2-A B P}^{N E}}{C_{2-A B P}^{a q}}$

where the equilibrium constant (or, partition coefficient) is $P, C_{2-A B P}^{N E}$ is the concentration of 2ABP partitioned into NE, $C_{2-A B P}^{a q}$ is the concentration of $2-\mathrm{ABP}$ remaining in aqueous phase.

According to the law of mass conservation,

$n_{2-A B P}^{t o t}=n_{2-A B P}^{N E}+n_{2-A B P}^{a q}$

where $n_{2-A B P}^{t o t}, n_{2-A B P}^{N E}$, and $n_{2-A B P}^{a q}$ are the number of moles of 2-ABP in the total system, in the NE phase, and remaining in the aqueous phase, respectively.

$\mathrm{Eq}$ (4) can be reformulated with concentrations of 2-ABP and volumes of the corresponding phases as below,

$C_{2-A B P}^{t o t} V_{t o t}=C_{2-A B P}^{N E} V_{N E}+C_{2-A B P}^{a q} V_{a q}$

Using eq (3) and eq (5), an implicit equation relating $C_{2-A B P}^{N E}$ with $C_{2-A B P}^{t o t}$ is obtained as below,

$C_{2-A B P}^{N E}=\left[\frac{V_{\text {total }}}{\frac{V_{a q}}{P}+V_{N E}}\right] \cdot C_{2-A B P}^{t o t}$

This relationship, eq (6) is readily used to extract the partition coefficient, $P$ with experimentally estimated $C_{2-A B P}^{N E}$ from the readout of SEE measurements under a series of various $C_{2-A B P}^{\text {tot }}$. Using three slopes in Figure 4, the respective experimental values (i.e. $V_{t o t a l}, V_{a q}$, and $V_{N E}$ ), and eq (6), the partition coefficient, $P$ could be determined as $1.9( \pm 1.4) \times 10^{10}$ (detailed parameters of $V_{\text {total }}$, $V_{a q}$, and $V_{N E}$ are in Table S1 in SI). In fact, one could evaluate a $P$ from one point measurement of SEE under a single condition, thus estimating $C_{2-A B P}^{N E}$ value and the corresponding $C_{2-A B P}^{a q}$ mathematically calculated by eq (3) and eq (5). This one point measurement, however, provides a 
$P$ with low accuracy. Contrarily, sets of measurements enable to construct an analogy of calibration curves and improve accuracy and precision to determine the $P$, thus attaining the $P$ value consistent over wide concentration ranges of 2-ABP as well as NEs.

The $P$ determined by SEE measurements is $\sim 7$ orders of magnitude higher than the reported partition coefficient of 2-ABP between two bulk phases, octanol and water $(P=691.8){ }^{20}$ Considering similar level of dielectric constants of two organic solvents, octanol and DOS, this $P$ determined for NEs is quite surprising. Owing to this unprecedentedly large $P$, the extraction by NEs is strikingly efficient with $\sim 8$ orders of magnitude of the preconcentration factor. This exceptional $P$ implies an additional stabilization of partitioned 2-ABP via the intermolecular interaction between 2-ABP and DOS in NEs. Indeed, the equilibrium constant (e.g. molecular recognition, binding, or catalysis) are often mediated by non-covalent interaction involving aromatic functional groups. ${ }^{23}$ Particularly, lone pair- $\pi$ (or referred to as $\mathrm{n}$ to $\pi^{*}$ ) interaction is ascribed to such a stabilizing association between a lone pair of electrons and the face of a $\pi$

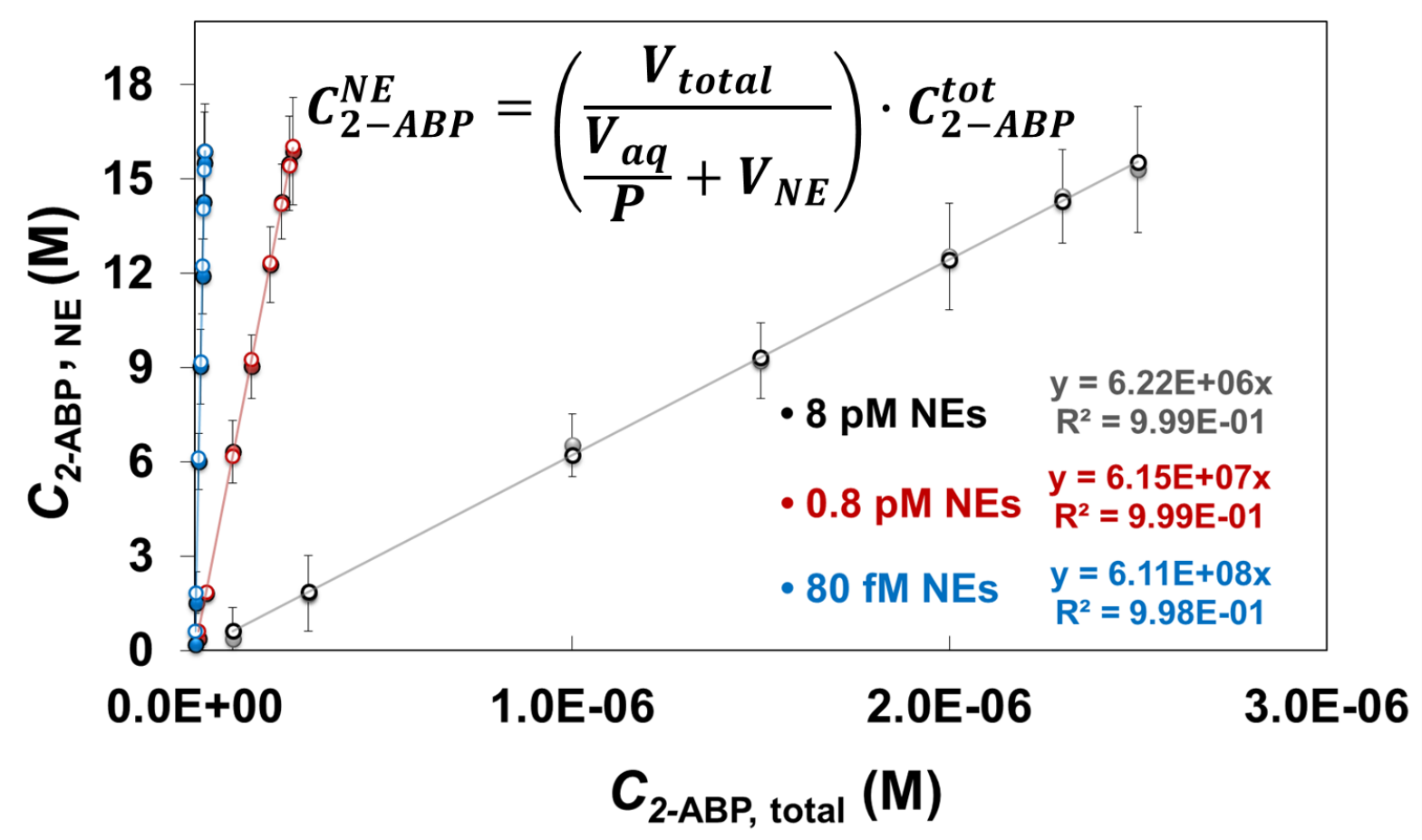

Figure 4. A plot of $\boldsymbol{C}_{2-A B P}^{N E}$ vs. $\boldsymbol{C}_{2-A B P}^{t o t}$ in the presence of various concentration of NEs, $8 \mathrm{pM}$ (black closed circles), $0.8 \mathrm{pM}$ (red closed circles), and $80 \mathrm{fM}$ (blue closed circles). The respective slopes and squared correlation coefficients, $R^{2}$ from the least square regression are shown in the bottom right. Each error bar represents the standard deviation from 1500 - 2000 current spikes under the given condition. 
system. ${ }^{23}$ Although it is expected to be quite weak, the significance of the lone pair- $\pi$ interaction has been noted along with other non-covalent interaction such as hydrogen-bonding. ${ }^{24,25}$ This interaction energy values are attractive and moderately strong, ranging from -11.3 to -94.5 $\mathrm{kJ} / \mathrm{mol}^{26}$

In our partitioned system, the carbonyl oxygen of DOS is in close proximity to the aromatic centers of 2-ABP, thereby the interaction between regions of negative (the lone pair) and positive (electron deficient $\pi$ system on the aromatic ring) can be understood as electrostatic potential (Figure 5). We performed the MD simulation using Spartan (Wavefunction Inc., Irvine, California, USA, 2014, 1.1.8 version), and calculated the interaction between 2-ABP and DOS molecule (see section 8 and Table S2 in SI). Based on our MD simulation, the interactions from these contacts are found to be favorable and stable with $\Delta G^{\circ}=-11.7 \mathrm{~kJ} / \mathrm{mol}$. To incorporate this stabilization energy into apparent partition coefficient, we consider a partitioning process of 2-ABP into NEs as two consecutive reactions, where 2-ABP partitioned into a NE undergoes a subsequent complexation with DOS molecules via non-covalent interaction.

$2-A B P(a q) \rightleftarrows 2-A B P(N E)$

$p 2-A B P(N E)+q D O S(N E) \rightarrow(2-A B P)_{p} \cdot(D O S(N E))_{q}$

where the equilibrium constant, $K$ for eq (7) is expressed by,

$$
K=\frac{C_{2-A B P, N E}}{C_{2-A B P, a q}}=\sim 691.8
$$

and the complex formation constant, $\beta$ for eq (8) is

$$
\beta=\frac{C_{2-A B P \cdot D O S}}{C_{2-A B P, N E}{ }^{p} \cdot C_{D O S}^{q}} \approx \frac{C_{2-A B P \cdot D O S}}{C_{2-A B P, N E}{ }^{p}}=\exp \left(-\Delta G^{0} / R T\right)
$$

where DOS is a pure solvent in NE phase, thus a concentration of DOS, $C_{D O S}$ is considered as unity.

Thereby, the apparent partition coefficient can be expressed by,

$P_{\text {app }}=\frac{\text { total concentration of } 2-A B P \text { in } N E}{\text { total concentration of } 2-A B P \text { in } a q}=K \cdot \beta$ 
To obtain $P_{a p p}$ consistent with the experimentally determined $P\left(=1.9( \pm 1.4) \times 10^{10}\right), \beta$ should be at least $3.0 \times 10^{7}$. Using eq (10) and (11), the anticipated $\Delta G^{\circ}$ for the stabilized complex in NEs would be $-42.6 \mathrm{~kJ} / \mathrm{mol}$. Although it is not clear to know the exact stoichiometry ratio of $p / q$, this anticipated $\Delta G^{\circ}$ is in the reasonable range between -11.3 and $-94.5 \mathrm{~kJ} / \mathrm{mol} .{ }^{26}$ Since $1: 1$ interaction between 2-ABP and DOS molecule results in $\Delta G^{\circ}=-11.7 \mathrm{~kJ} / \mathrm{mol}$, a collective contribution from the interaction between multiple molecules could be considered rationally (see Table S3 in SI). Therefore, our calculations support the idea that the intermolecular interaction between the carbonyl oxygens in DOS and the aromatic center of 2-ABP as well as hydrogen bonding could play a stabilizing role in partitioned structures inside NEs, thus leading to a remarkably high partition coefficient.

(A)

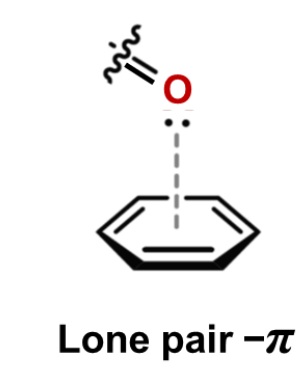

(B)

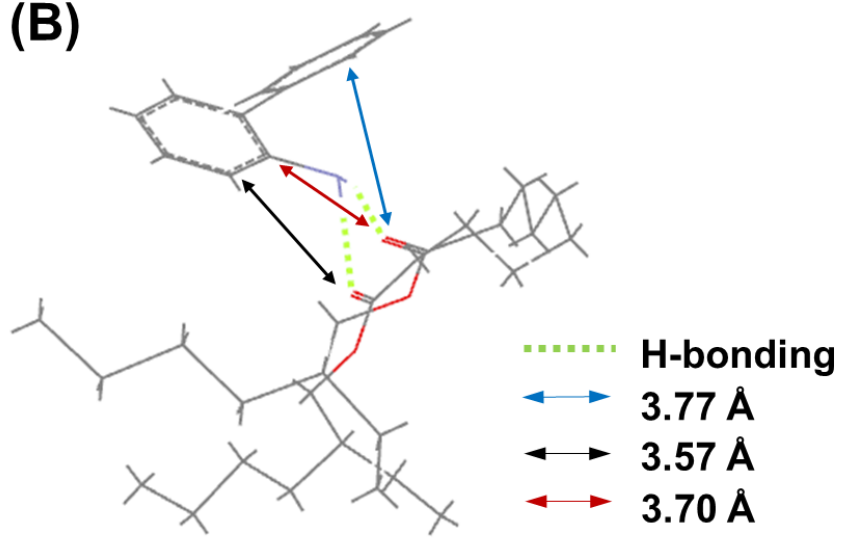

Figure 5. (A) Interaction of lone pair and $\pi$ system. (B) Geometry optimized structure of 2-ABP with DOS molecule: lone pair- $\pi$ interaction (red, blue and black arrows) with hydrogen bonding (green dotted line).

We further verified our methodology with other compounds exhibiting aromatic property as well as hydroxyl moiety for hydrogen bonding formation, i.e., FcMeOH. The determined $P_{a p p}$ of FcMeOH at intact NEs was $2.6( \pm 1.6) \times 10^{9}$ throughout both in situ SEE measurements and a constructed linear plot of $\boldsymbol{C}_{\boldsymbol{F c M e O H}}^{N E}$ vs. $\boldsymbol{C}_{\text {FcMeOH }}^{\text {total }}$, (Figure S6 and S7 in SI). This $P_{\text {app }}$ is also $\sim 7$ orders of magnitude higher than the reported partition coefficient of $\mathrm{FcMeOH}$ between two bulk phase, 1,2-dichlorobenzene and water $(P=82)$ (or, $K$ in eq (9)) ${ }^{27}$ This result is consistent with the case of 2-ABP with supporting the intermolecular interactions between $\pi^{*}$ of aromatic ring and 
the lone pair in DOS as well as the hydrogen bonding inside NEs, confirmed by the MD simulations (See Figure S8, section 7 and 8 in SI,).

\section{Conclusions}

In conclusions, we could successfully employ SEE to in situ measure the partition coefficient of target compounds at intact NEs, thus exhibiting a practical insight in SEE. The direct and in-situ measurements of extracted 2-ABP from water to Pluronic F-127 functionalized NEs were enabled by the instantaneous electrochemical oxidation upon the collision of each individual NE onto Pt UME. The unprecedentedly large preconcentration factor as $\sim 8$ orders of magnitude could be obtained, thus resulting in an apparent partition coefficient of $1.9( \pm 1.4) \times 10^{10}$. This large partition coefficient could be attributed to the intermolecular interaction in NEs, which was quantitatively validated by the MD simulations. It should be noted that the high monodispersity of NEs in this study allows for high precision and accuracy in our measurements. Significantly, our approach is readily applicable to investigate practical NEs commercially available for drug, food, and cosmetics, and to develop ultrasensitive environmental or biomedical analysis using NEs. ${ }^{28}$

\section{Author Information}

\section{Corresponding Author}

*Phone: +1 (401) 874-2143. E-mail: jkim25@ uri.edu.

\section{ORCID}

Jiyeon Kim: 0000-0002-7624-6766

Shashika Gunathilaka Gunathilaka Sabaragamuwe: 0000-0002-5000-8559

\section{Author Contributions}

All authors have given approval to the final version of the manuscript

\section{Notes}

The authors declare no competing financial interest.

\section{Supporting Information}

Experimental Section, Characterization of NEs by Dynamic Light Scattering, Electrochemistry of 2-ABP in THF Cocktail Solution, Nanopipet Voltammetry of $\mathrm{PF}_{6}^{-}$Ion Transfer across the 
Immiscible Interface between DOS and Aqueous Buffer, Fitting $i$ - $t$ Decay with Bulk Electrolysis Model, Additional SEE data for 2-ABP Partitioned at Intact NEs, SEE Data and In Situ Measurement of Partition Coefficient of FcMeOH at Intact NEs, and Molecular Dynamics Simulation Results.

\section{Acknowledgements}

This work was supported by the Research Bridge Funding Initiative, Start up fund from University of Rhode Island, Medical Research Fund (20174373) from Rhode Island Foundation, and in part by the Rhode Island Institutional Development Award (IDeA) Network of Biomedical Research Excellence from the National Institute of General Medical Sciences of the National Institutes of Health (P20GM103430). We acknowledge Irene Andreu for TEM characterization conducted at the RI Consortium for Nanoscience and Nanotechnology, supported by the National Science Foundation EPSCoR Cooperative Agreement \#OIA-1655221 and the College of Engineering at URI.

\section{References}

1. Lovelnm C.; Attama, A. A., "Current state of Nanoemulsions in Drug Delivery", J. Biomater. Nanobiotechnol., 2011, 2, 626-639.

2. Tadros, T.; Izquierdo, P.; Esquena, J.; Solans, C., "Formation and Stability of Nanoemulsions", Adv. Colloid. Interfac. Sci., 2004, V108-109, 303-318.

3. Abooofazeli, R., "Nanometric Scaled Emulsions (Nanoemulsions)", Iran. J. Pharm. Res., 2010, 9, 325-326.

4. Lucy, C. A.; Harris, D. C., "Quantitative Chemical Analysis", 2016, $9^{\text {th }}$ Ed., W. H. Freeman and Company.

5. Ganra, S.; Talejar, M.; Singh, A.; Coleman, T. P.; Amiji, M. M., "Nanoemulsions in Translational Research - Opportunities and Challenges in Targeted Cancer Therapy", AAPS PharmaSciTech, 2014, 15, 694-708.

6. Lallenmad, F.; Daull, P.; Benita, S.; Buggage, R.; Garrigue, J-S., "Successfully Improving Ocular Drug Delivery Using the Cationic Nanoemulsion, Novasorb", J. Drug Delivery, 2012, 116.

7. Varshney, M.; Morey, T. E.; Shah, D. O.; Flint, J. A.; Moudgil, B. M.; Seubert, C. N.; Dennis, D. M., "Pluronic Microemulsions as Nanoreserviors for Extraction of Bupivacaine from Normal Saline", J. Am. Chem. Soc., 2004, 126, 5108-5112.

8. Liu, C-H.; Lai, K-Y.; Chen, Y-J.; Lee, W-S.; Hsu, C-Y., "In Vitro Scleral Lutein Distribution by Cyclodextrin Containing Nanoemulsions", Chem. Pharm. Bull., 2015, 63, 59-67.

9. Singh, K. K.; Vingkar, S. K., "Formulation, Antimalarial Activity and Biodistribution of Oral Lipid Nanoemulsion of Primaquine”, Int. J. Pharm., 2008, 347, 136-143. 
10. Ahmad J, F.; Ali, M.; Shekel, F.; Talegaonakr, C.; Khar K, R.; Shafig, S., "Investigation of Nanoemulsion System for Transdermal Delivery of Domperidone: Ex-vivo and in vivo Studies", 2008, Current Nanoscience, 4, 381-390.

11. Srilatha, R.; Aparna, C.; Srinivas, P.; Sadanandam, M., "Formulation, Evaluation and Characterization of Glipizide Nanoemulsions", Asian J. Pharm. Clin. Res., 2013, 6, 66-71.

12 . Bhagav, P.; Upadhay, H.; Chandra, S., "Brimonidine Tartrate Eudragit Long-acting Nanoparticle: Formulation, Optimization, in vitro and in vivo Evaluation", AAPS PharmSciTech, 2011, 12, 1087-1101.

13. Barker, A. L.; Macpherson, J. V.; Slevin, C. J.; Unwin, P. R., "Scanning Electrochemical Microscopy (SECM) as a Probe of Transfer Processes in Two-Phase Systems: Theory and Experimental Applications of SECM-Induced Transfer with Arbitrary Partition Coefficients, Diffusion Coefficients, and Interfacial Kinetics", J. Phys. Chem. B, 1998, 102, 1586-1598.

14 . Knotturi, K.; Murtomäki, L., "Electrochemical Determination of Partition Coefficients of Drugs", J. Pharmaceutical Sci., 1992, 81, 970-975.

15. Ascendiapharma, https://ascendiapharma.com/technologies/emulsol/

16 . Sabaragamuwe, S. G.; Conti, D.; Puri, S. R.; Andreu, I.; Kim, J, "Single-Entity Electrochemistry of Nanoemulsions: The Nanostructrual Effect on Its Electrochemical Behavior", Anal. Chem., 2019, 91, 9599-9607.

17 . Kim, B-K.; Boika, A.; Kim, J.; Dick, J. E.; Bard. A. J.," Characterizing Emulsions by Observation of Single Droplet Collisions: Attoliter Electrochemical Reactors", J. Am. Chem. Soc., 2014, 136, 4849-4852.

18 . Xie, X.; Zhai, J.; Crespo, G.; Bakker, E., "Ionophore-Based Ion Sensitive Optical NanoSensors Operating in Exhaustive Sensing Mode", Anal. Chem., 2014, 86, 8770-8775.

19. Xie, X.; Zhai, J.; Bakker, E., "pH Independent Nano-Optode Sensors Based on Exhaustive Ion-Selective Nanospheres", Anal. Chem., 2014, 86, 2853-2856.

20 . Hansch, C., Leo, A., D. Hoekman., "Exploring QSAR - Hydrophobic, Electronic, and Steric Constants.”, Washington, DC: American Chemical Society., 1995, p. 98

21 R. N. Adams, "Electrochemistry at Solid Electrodes", 1969, Marcel Dekker, New York, NY.

22. V. Vyskocil, J. Barek, "Electroanalysis of nitro and amino derivatives of polycyclic aromatic hydrocarbons", Curr. Org. Chem., 2011, 15, 3059-3076.

23. Neel, A. J.; Hilton, M. J.; Sigman, M. S.; Tosste, F. D., "Exploiting Non-Covalent $\pi$ Interactions for Catalyst Design", Nature, 2017, 543, 637-646.

24 . Singh, S. K.; Kumar, S.; Das, A., "Competition between $n \rightarrow \pi(A r) *$ and Conventional Hydrogen Bonding $(\mathrm{N}-\mathrm{H} \cdots \mathrm{H})$ Interactions: an ab initio Study of the Complexes of 7-azaindole and fluorosubstituted pyridines", Phys. Chem. Chem. Phys., 2014, 16, 8819-8827.

25. Ao, M-Z.; Tao, Z-Q.; Liu, H-X.; Wu, D-Y.; Wang, X., "A Theoretical Investigation of the Competition between Hydrogen Bonding and Lone Pair $\cdots \pi$ Interaction in Complexes of TNT with $\mathrm{NH}_{3}$ ", Comput. Theor. Chem., 2015, 1064, 25-34.

26. Frontera, A.; Bauzá, A., "Concurrent Aerogen Bonding and Lone Pair/Anion- $\pi$ Interactions in the Stability of Organoxenon Derivatives: a Combined CSD and ab initio Study", Phys. Chem. Chem. Phys., 2017, 19, 30063-30068.

27. Peljo, P.; Qiao, L.; Murtomäki, L.; Johans, C.; Girault, H. H.; Kontturi, K., "Electrochemically Controlled Proton-Transfer-Catalyzed Reactions at Liquid-Liquid Interfaces: Nucleophilic Substitution on Ferrocene Methanol", Chem. Phys. Chem., 2013, 14, 311-314. 
28. Sabaragamuwe, S. G.; Madawala, H.; Puri, S. R.; Kim, J., "Towards Ultralow Detection Limits of Aromatic Toxicants in Water Using Pluronic Nanoemulsions and Single-entity Electrochemistry", Anal. Chim. Acta., 2020, 1139, 129-137.

For TOC only

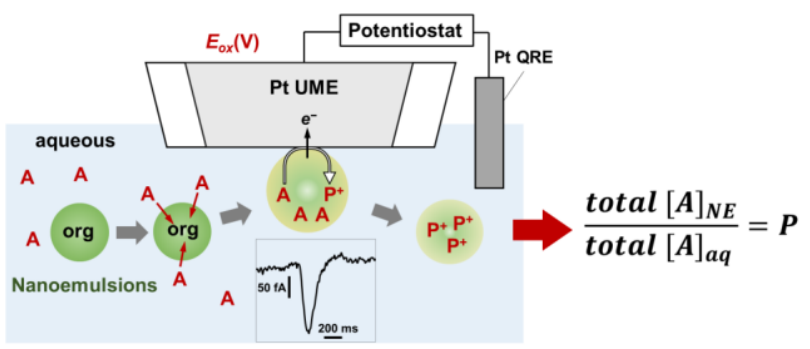

